\title{
Prevalência, perfil e fatores associados à automedicação em adolescentes e servidores de uma escola pública profissionalizante
}

\author{
Prevalence, profile and factors associated with \\ self-medication in adolescents and employees of a professionalizing \\ public school
}

\author{
Januária Fonseca Matos ${ }^{1}$, Davi Alexander Costa Pena¹, Milena Pereira Parreira', \\ Tamires do Carmo dos Santos ${ }^{1}$, Wendel Coura-Vital ${ }^{2}$
}

\begin{abstract}
Resumo
Objetivos: Avaliar a prevalência, o perfil e fatores associados à automedicação na população do Instituto Federal Minas Gerais/Ouro Preto, constituída principalmente por adolescentes. Método: Trata-se de um estudo transversal, no qual foi aplicado um questionário aos alunos e servidores da Instituição abordando questões sobre nível socioeconômico, características gerais, condição de saúde autorreferida, medicamento utilizado nos últimos 15 dias, uso de medicamento com e sem receita médica e questões relativas à automedicação. A regressão de Poisson foi empregada para avaliar os fatores associados à automedicação. Resultados: Foram entrevistadas 270 pessoas, sendo 231 alunos e 39 servidores. A prevalência de automedicação foi de $69,3 \%$ (IC95\% 63,6-74,6), sendo os analgésicos a principal classe farmacológica utilizada. Os fatores associados à automedicação foram: utilizar medicamento influenciado por propaganda $(\mathrm{RP}=1,2 \mathrm{IC} 95 \%=1,1-1,4)$; a prática de indicar medicamento $(\mathrm{RP}=1,4 \mathrm{IC} 95 \%=1,1-1,6)$; estado de saúde autorreferido muito bom/bom ( $\mathrm{RP}=0,8 \mathrm{IC} 95 \%=0,6-0,9)$ e ter realizado a última consulta médica há mais de um mês ( $\mathrm{RP}=1,5 \mathrm{IC} 95 \%=1,1-2,1)$. Conclusão: Estes fatores reforçam a importância do acesso a consultas médicas e de ações de conscientização sobre o uso racional de medicamentos.
\end{abstract}

Palavras-chave: automedicação; adolescentes; fatores associados.

\begin{abstract}
Objectives: To evaluate the prevalence, profile and factors associated with self-medication in the population of the Federal Institute of Minas Gerais (Instituto Federal de Minas Gerais) - Ouro Preto. Method: This is a cross-sectional study, in which a questionnaire was applied to students and staff of the Institution addressing issues such as socioeconomic status, general characteristics, self-reported health condition, medication used in the previous 15 days, use of prescription and over-the-counter medication. Poisson regression was used to evaluate the factors associated with self-medication. Results: 270 individuals were interviewed, 231 students and 39 employees. The prevalence of self-medication was $69.3 \%(95 \% \mathrm{Cl} 63.6-74.6)$, analgesics being the main pharmacological class used. The factors associated with self-medication were: to use medicine influenced by advertising $(\mathrm{PR}=1.2 ; 95 \% \mathrm{Cl}=1.1-1.4)$; the practice of indicating medication $(P R=1.4 ; 95 \% \mathrm{Cl}=1.1-1.6)$; self-reported health status very good $/$ good $(P R=0.8 ; 95 \% \mathrm{Cl}=0.6-0.9)$, and having the last medical appointment for more than a month $(\mathrm{RP}=1.5 ; 95 \% \mathrm{Cl}=1.1-2.1)$. Conclusion: These factors reinforce the importance of access to medical consultations and awareness actions of the rational use of medicines.

Keywords: self-medication; adolescents; associated factors.

${ }^{1}$ Instituto Federal Minas Gerais (IFMG), Campus Ouro Preto - Ouro Preto (MG), Brasil.

2Departamento de Análises Clínicas, Escola de Farmácia, Universidade Federal de Ouro Preto (UFOP) - Ouro Preto (MG), Brasil.

Trabalho realizado no Instituto Federal Minas Gerais (IFMG) - Ouro Preto (MG), Brasil.

Endereço para correspondência: Januária Fonseca Matos - Instituto Federal Minas Gerais (IFMG), Campus Ouro Preto, Rua Pandiá Calógeras, 898 -

Bauxita - CEP: 35400-000 - Ouro Preto (MG), Brasil - Email: januaria.matos@ifmg.edu.br

Fonte de financiamento: nenhuma.

Conflito de interesses: nada a declarar.
\end{abstract}

Este é um artigo publicado em acesso aberto (Open Access) sob a licença Creative Commons Attribution, que permite uso, distribuição e reprodução em qualquer meio, sem restrições desde que o trabalho original seja corretamente citado. 


\section{INTRODUÇÃO}

A automedicação é uma prática caracterizada fundamentalmente pela iniciativa de um doente, ou de seu responsável, em obter ou produzir e utilizar um produto que acredita lhe trazer benefícios no tratamento da doença ou alívio de sintomas ${ }^{1}$. Assim, a orientação médica é substituída inadvertidamente por sugestões de medicamentos provenientes de pessoas não autorizadas, entre estas familiares, amigos ou balconistas em farmácias. Outra forma de automedicação seria a reutilização de receitas médicas anteriores, mesmo que o uso contínuo do medicamento não tenha sido especificado ${ }^{1,2}$. No Brasil, o Sistema Nacional de Informações Tóxico-Farmacológicas (Sinitox/Fiocruz) registrou, só em 2011, cerca de 30 mil casos de intoxicação por uso de medicamentos e 53 mortes, correspondendo a uma letalidade de $0,18 \%$. Os medicamentos foram a primeira causa de intoxicação humana por agente tóxico, sendo responsável por $28,6 \%$ do total de casos registrados deste tipo de intoxicação. Crianças menores de 5 anos representam, aproximadamente, $30 \%$ dos casos de intoxicação por medicamentos, no Brasil ${ }^{3}$. Em 2013, foram registrados cerca 12 mil casos de intoxicação por medicamentos e 46 mortes, sendo verificado um aumento da letalidade $(0,38 \%)$ comparado ao ano de $2011^{3}$. De acordo com dados da Organização Mundial da Saúde (OMS), os hospitais gastam $15 \%$ a $20 \%$ de seus orçamentos para lidar com as complicações causadas pela automedicação ${ }^{4}$.

Os riscos da automedicação para o indivíduo são o atraso no diagnóstico ou o diagnóstico incorreto, devido ao mascaramento dos sintomas, possibilitando o agravamento do distúrbio; a escolha do medicamento inadequado; a administração incorreta, dosagem inadequada e uso excessivamente curto ou prolongado do medicamento; a dependência; a possibilidade da ocorrência de efeitos indesejados graves; o desconhecimento das interações com outros medicamentos; reações alérgicas, intoxicações; e, ainda, o armazenamento incorreto e uso do medicamento fora de seu prazo de validade ${ }^{5,6}$. Além do impacto sobre a vida humana, as reações adversas a medicamentos também influenciam significativamente nos custos despendidos com saúde ${ }^{7}$.

Diversos trabalhos avaliando a automedicação observaram que alguns dos fatores que influenciam os indivíduos a se automedicarem são: a dificuldade de acesso aos serviços de saúde, tanto pela demora quanto pelo preço de uma consulta médica; a limitação do poder prescritivo; a recomendação de medicamentos por conhecidos ou balconistas de farmácias; a repetição de sintomas anteriores, levando o indivíduo a seguir as prescrições já utilizadas; a falta de tempo para procurar um profissional da saúde; a divulgação de medicamentos pela mídia e o livre comércio de medicamentos, passando a imagem de que são produtos inofensivos à saúde $\mathrm{e}^{7-15}$.

Os adolescentes são um grupo vulnerável da população em relação ao uso de medicamentos uma vez que nessa fase é iniciada a utilização de anticoncepcionais, anorexígenos para a perda de peso e anabolizantes, que são relacionados à perfeição corporal cobrada pela sociedade atual ${ }^{7,16,17}$. Apesar desta vulnerabilidade, ainda são escassos os estudos que avaliam a automedicação nesta população ${ }^{18-20}$. Segundo o Ministério da Saúde brasileiro, em 2012 foram notificados 2.243 casos de intoxicação por medicamento entre pré-adolescentes (10 a 14 anos) e adolescentes (15-19 anos), o que corresponde a $15,7 \%$ do total de casos na região Sudeste do Brasil. Além disso, foram registrados em 201239 óbitos de intoxicação por medicamento na região Sudeste, sendo sete (18\%) desses entre pré-adolescentes e adolescentes ${ }^{3}$. Diante do exposto, o objetivo deste estudo foi avaliar a prevalência, o perfil da automedicação e os fatores associados a esta prática em uma escola técnica profissionalizante, cuja população é constituída em sua maioria por adolescentes.

\section{MÉTODO}

\section{Aspectos éticos}

Este estudo foi aprovado pelo Comitê de Ética em Pesquisa com Seres Humanos CEP/IF SUDESTE MG, sob o número 1.129.341. Os indivíduos foram informados sobre o objetivo da pesquisa, e aqueles que aceitaram participar assinaram o Termo de Consentimento Livre e Esclarecido (TCLE). Caso o entrevistado fosse menor de idade, este assinou o Termo de Assentimento Livre e Esclarecido (TALE) e seus responsáveis, o TCLE.

\section{Desenho do estudo}

Foi realizado um estudo transversal no período compreendido entre 2015 e 2016. Durante o estudo foi aplicado um questionário aos alunos, professores e demais servidores do Instituto Federal Minas Gerais (IFMG), Campus Ouro Preto. O IFMG Campus Ouro Preto possui ensino técnico integrado ao ensino médio, ensino à distância e cursos de graduação (tecnólogo e licenciatura), totalizando aproximadamente 2.450 alunos, e conta com 432 servidores (docentes e técnicos administrativos). Para o cálculo amostral, considerou-se uma prevalência de uso global de medicamentos de aproximadamente $65-75 \%{ }^{2,21}$, uma população de 2.880 indivíduos, nível de confiança de 95\% e margem de erro de $5 \%$, resultando em aproximadamente 270 entrevistas, sendo proporcional o número de alunos e servidores. O critério de inclusão para a participação no trabalho foi ter utilizado medicamentos prescritos e/ou não prescritos nos últimos 15 dias que antecederam a pesquisa.

As entrevistas foram realizadas com os alunos durante os intervalos entre as aulas, na cantina, na biblioteca e em outros espaços de convívio. Os servidores foram entrevistados em seus locais de trabalho. Foi mantida a proporção de entrevistas entre servidores e alunos da instituição. 


\section{Coleta de dados}

As perguntas contidas no questionário abordaram questões sobre nível socioeconômico do entrevistado, características gerais da população, condição de saúde autorreferida, medicamentos utilizados nos últimos 15 dias, número de consultas médicas nos últimos 12 meses, uso de medicamentos com e sem receita médica, justificativa para o uso do medicamento sem receita médica, responsáveis pela indicação do medicamento alvo de automedicação, uso de medicamentos concomitantes à automedicação, reação adversa e satisfação com o uso do medicamento não prescrito, influência dos meios de comunicação, confiança nas propagandas de medicamentos, leitura da bula e classes farmacológicas mais utilizadas. O nível socioeconômico foi avaliado através do Critério de Classificação Econômica Brasil 201022, que leva em consideração o grau de instrução do chefe da família e a presença de itens domésticos na residência.

\section{Análise estatística}

Os dados obtidos através da aplicação dos questionários foram duplamente digitados no software EpiData 3.1 e, após correção das divergências de digitação, foram exportados e analisados no software Stata 10. Foi calculada a prevalência da automedicação, sendo que esta se refere à proporção de indivíduos que se automedicaram entre pessoas que tomaram medicamentos nos últimos 15 dias. Para investigar os fatores associados à automedicação, os participantes do estudo foram divididos em dois grupos, um deles composto por indivíduos que se automedicaram e o outro pelos que não se automedicaram. O critério para caracterizar o indivíduo como pertencente ao grupo automedicação foi ter consumido medicamentos sem prescrição médica nos últimos 15 dias em concomitância ou não com o uso de medicamentos prescritos. A regressão de Poisson foi empregada para avaliar os fatores associados à automedicação. Inicialmente foi realizada uma análise univariada avaliando todas as variáveis contidas no questionário. As variáveis que apresentaram valor de $\mathrm{p}<0,25$ foram selecionadas para a análise multivariada. Variáveis com mais de duas categorias foram transformadas em variáveis dummies. $\mathrm{Na}$ análise multivariada utilizou-se o nível de significância de 0,05 , isto é, as variáveis que apresentaram $\mathrm{p}>0,05$ foram retiradas do modelo passo a passo (backward) e foram mantidas no modelo final apenas aquelas com nível de significância de p<0,05.

\section{RESULTADOS}

\section{Características da população}

Participaram do estudo 270 indivíduos (181 adolescentes e 89 adultos), com idade média de 23,1 $\pm 10,8$; sendo a mínima 15 anos e a máxima 70 anos. A maior parcela dos entrevistados corresponde a alunos $(85,6 \%)$. A maioria foi do sexo feminino $(\mathrm{n}=158 ; 58,5 \%)$ e 231 (85,6\%) são solteiros. Quanto à escolaridade do responsável, foi observado que a maioria tem ensino médio (mães $n=101 ; 37,4 \%$; pais n=108; 40\%). Com relação às condições socioeconômicas, segundo o Critério de Classificação Econômica Brasil, observou-se que 99 (36,7\%) são da classe social B2, sendo a renda do chefe de família de aproximadamente $\mathrm{R} \$ 3.000,00$. Em relação a indicadores de saúde, mais da metade das pessoas entrevistadas não são fumantes $(\mathrm{n}=239 ; 88,5 \%)$ e pratica exercícios entre 1 e 3 vezes semanais ( $\mathrm{n}=122 ; 45,2 \%)$. A maioria da população analisada $(n=155 ; 57,4 \%)$ possui plano de saúde privado (Tabela 1). Em relação ao uso de medicamento, um fato interessante observado é que embora 70,7\% $(n=191)$ dos participantes considerem as propagandas de medicamentos pouco ou nada confiáveis, quase metade ( $\mathrm{n}=122 ; 45,2 \%)$ utilizou medicamentos por influência delas. Quando questionados se indicariam o medicamento utilizado (prescrito ou não prescrito) para outra pessoa, $173(64,1 \%)$ responderam que indicariam. Interessantemente, 185 (68,5\%) dos entrevistados afirmaram ler as bulas dos medicamentos, entretanto, 247 (91,5\%) acham esta prática importante. Quanto à percepção da própria saúde, a maioria considera muito boa ou boa $(\mathrm{n}=175 ; 64,8 \%)$ (Tabela 2$)$.

\section{Prevalência e perfil da automedicação}

Foi observada uma elevada proporção de indivíduos que se automedicaram entre pessoas que tomaram medicamentos nos últimos 15 dias na população avaliada: 69,3\% (IC95\%: 63,6-74,6). $\mathrm{E}$ os medicamentos não prescritos mais utilizados foram os analgésicos/antitérmicos $(\mathrm{n}=123 ; 65,8 \%)$, seguidos pelos antigripais $(21,4 \%)$ e os anti-inflamatórios (18,7\%). O sintoma responsável pela maior parte dos casos de automedicação foi a comum dor de cabeça/febre (67,9\%), seguido por resfriado/gripe (32,6\%), e o consumo do medicamento não prescrito ocorreu em $36,9 \%$ das vezes, por conta própria, seguido pela orientação da mãe $(32,1 \%)$ (Tabela 3). O motivo mais apontado para a prática da automedicação foi a experiência anterior com o medicamento utilizado (54,5\%) (Tabela 4). Embora praticamente a totalidade de entrevistados não apresentar reação adversa $(95,2 \%)$ e estar satisfeita $(94,1 \%)$ com o uso do medicamento não prescrito, foi possível observar que $51,9 \%$ não sabiam se tais medicamentos poderiam lhes causar algum problema. Foi observado também que 35 (18,7\%) dos indivíduos que se automedicaram estavam utilizando outro medicamento por ocasião da automedicação (Tabela 3).

\section{Fatores associados à automedicação}

A comparação entre os indivíduos que se automedicaram e os que não realizaram esta prática foi previamente realizada através da análise univariada, e os resultados estão apresentados 
Tabela 1. Análise bivariada da automedicação e características gerais da população, Ouro Preto, 2015/2016

\begin{tabular}{|c|c|c|c|c|}
\hline \multirow{2}{*}{ Variáveis } & \multicolumn{2}{|c|}{ Automedicação } & \multirow{2}{*}{$\begin{array}{c}\text { RP (IC } \\
95 \%)\end{array}$} & \multirow{2}{*}{$\mathbf{p}$} \\
\hline & $\operatorname{Sim}(\%)$ & Não (\%) & & \\
\hline \multicolumn{5}{|l|}{ Grupo etário } \\
\hline Adolescentes & $125(66,8)$ & $56(67,5)$ & 1 & \\
\hline Adultos & $62(33,2)$ & $27(32,5)$ & $1.0(0,8-1,2)$ & 0,92 \\
\hline \multicolumn{5}{|l|}{ Categoria funcional } \\
\hline Discente & $165(88,2)$ & $66(79,5)$ & 1 & \\
\hline Servidor & $22(11,8)$ & $17(20,5)$ & $1,3(0,9-1,7)$ & 0,11 \\
\hline \multicolumn{5}{|l|}{ Sexo } \\
\hline Masculino & $74(39,6)$ & $38(45,8)$ & 1 & \\
\hline Feminino & $113(60,4)$ & $45(54,2)$ & $1,1(0,9-1,3)$ & 0,35 \\
\hline \multicolumn{5}{|l|}{ Estado civil } \\
\hline Solteiro & $159(85,0)$ & $72(86,7)$ & 1 & \\
\hline Casado & $25(13,4)$ & $8(9,6)$ & $1,1(0,9-1,4)$ & 0,37 \\
\hline Divorciado & $3(1,6)$ & $3(3,6)$ & $0,7(0,3-1,6)$ & 0,44 \\
\hline \multicolumn{5}{|l|}{ Escolaridade pai } \\
\hline $\begin{array}{l}\text { Fundamental } \\
\text { completo }\end{array}$ & $67(35,8)$ & $25(30,1)$ & 1 & \\
\hline Ensino Médio & $76(40,6)$ & $32(38,6)$ & $1,0(0,8-1,1)$ & 0,70 \\
\hline $\begin{array}{l}\text { Graduação/ } \\
\text { Pós-Graduação }\end{array}$ & $44(23,5)$ & $26(31,3)$ & $0,9(0,7-1,1)$ & 0,19 \\
\hline \multicolumn{5}{|l|}{ Escolaridade mãe } \\
\hline $\begin{array}{l}\text { Fundamental } \\
\text { completo }\end{array}$ & $54(28,9)$ & $22(26,5)$ & 1 & \\
\hline Ensino Médio & $69(36,9)$ & $32(38,6)$ & $1,0(0,8-1,2)$ & 0,69 \\
\hline $\begin{array}{l}\text { Graduação/ } \\
\text { Pós-Graduação }\end{array}$ & $64(34,2)$ & $29(34,9)$ & $1,0(0,8-1,2)$ & 0,75 \\
\hline \multicolumn{5}{|l|}{ Classe social ${ }^{\star}$} \\
\hline $\mathrm{A} 1, \mathrm{~A} 2, \mathrm{~B} 1$ & $47(25,1)$ & $31(37,3)$ & 1 & \\
\hline B2 & $71(38,0)$ & $28(33,7)$ & $1,2(1,0-1,5)$ & 0,12 \\
\hline $\mathrm{C} 1, \mathrm{C} 2, \mathrm{D}, \mathrm{E}$ & $69(36,9)$ & $24(28,9)$ & $1,2(1,0-1,5)$ & 0,06 \\
\hline \multicolumn{5}{|l|}{ Tabagista } \\
\hline Não & $167(91,8)$ & $72(91,1)$ & 1 & \\
\hline Ex-fumante & $2(1,1)$ & $5(6,3)$ & $0,4(0,1-1,3)$ & 0,13 \\
\hline Sim & $13(7,1)$ & $2(2,5)$ & $1,2(1,0-1,5)$ & 0,05 \\
\hline \multicolumn{5}{|l|}{$\begin{array}{l}\text { Prática de exercícios } \\
\text { físicos }\end{array}$} \\
\hline Não pratico & $65(34,8)$ & $27(32,5)$ & 1 & \\
\hline 1 a 3 vezes semanais & $79(42,2)$ & $43(51,8)$ & $0,9(0,8-1,1)$ & 0,36 \\
\hline $\begin{array}{l}4 \text { vezes ou mais } \\
\text { semanais }\end{array}$ & $43(23,0)$ & $13(15,7)$ & $1,1(0,9-1,3)$ & 0,40 \\
\hline \multicolumn{5}{|l|}{$\begin{array}{l}\text { Possui plano de } \\
\text { saúde privado }\end{array}$} \\
\hline Não & $85(45,5)$ & $30(36,1)$ & 1 & \\
\hline Sim & $102(54,5)$ & $53(63,9)$ & $0,9(0,8-1,0)$ & 0,15 \\
\hline \multicolumn{5}{|l|}{$\begin{array}{l}\text { Última consulta } \\
\text { médica }\end{array}$} \\
\hline Na última semana & $19(10,2)$ & $18(21,7)$ & 1 & \\
\hline $\begin{array}{l}\text { Entre } 1 \text { semana e } \\
1 \text { mês }\end{array}$ & $29(15,5)$ & $32(38,5)$ & $0,9(0,6-1,4)$ & 0,71 \\
\hline Entre 1 e 3 meses & $69(36,9)$ & $18(21,7)$ & $1,5(1,1-2,2)$ & 0,01 \\
\hline Há mais de 3 meses & $70(37,4)$ & $15(18,1)$ & $1,6(1,2-2,2)$ & 0,01 \\
\hline
\end{tabular}

Tabela 2. Análise bivariada da automedicação e características da população em relação a medicamentos, Ouro Preto, 2015/2016

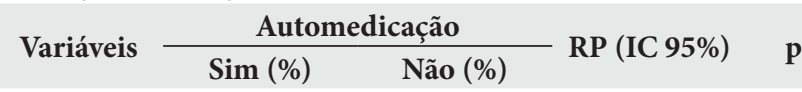

Confia nas propagandas de medicamentos

$\begin{array}{lcccc}\text { Nada } & 13(6,9) & 16(19,3) & 1 & \\ \text { Pouco } & 120(64,2) & 42(50,6) & 1,6(1,1-2,5) & 0,02 \\ \text { Muito } & 26(13,9) & 5(6,0) & 1,9(1,2-2,9) & 0,01 \\ \begin{array}{l}\text { Não sabe } \\ \text { responder }\end{array} & 28(15,0) & 20(24,1) & 1,3(0,8-2,1) & 0,27\end{array}$

Uso de medicamento influenciado por propaganda

$\begin{array}{lllcl}\text { Não } & 94(50,3) & 54(65,1) & 1 & \\ \text { Sim } & 93(49,7) & 29(34,9) & 1,2(1,1-1,4) & 0,02\end{array}$

Indicou ou indicaria algum medicamento

$\begin{array}{lrrcc}\text { Não } & 53(28,3) & 44(53,0) & 1 & \\ \begin{array}{l}\text { Sim } \\ \text { Lê bula }\end{array} & 134(71,7) & 39(47,0) & 1,4(1,2-1,7) & 0,00 \\ \text { Não } & 62(33,1) & 23(27,7) & 1 & \\ \begin{array}{l}\text { Sim, às } \\ \text { vezes }\end{array} & 94(50,3) & 44(53,0) & 0,9(0,8-1,1) & 0,44 \\ \begin{array}{l}\text { Sim, } \\ \text { sempre }\end{array} & 31(16,6) & 16(19,3) & 0,9(0,7-1,2) & 0,42\end{array}$

Considera leitura da bula importante

$\begin{array}{ccccc}\text { Não } & 16(8,6) & 7(8,4) & 1 & \\ \text { Sim } & 171(91,4) & 76(91,6) & 1,0(0,7-1,3) & 0,97\end{array}$

Percepção da própria saúde

\begin{tabular}{lcccc}
$\begin{array}{l}\text { Regular/ } \\
\text { Ruim }\end{array}$ & $44(23,5)$ & $11(13,3)$ & 1 & \\
$\begin{array}{l}\text { Muito } \\
\text { boa/boa }\end{array}$ & $111(59,4)$ & $64(77,1)$ & $0,8(0,7-0,9)$ & 0,01 \\
Excelente & $32(17,1)$ & $8(9,6)$ & $1,0(0,8-1,2)$ & 1,0 \\
\hline
\end{tabular}

nas Tabelas 1 e 2 . As variáveis que apresentaram nível de significância menor que 0,25 foram selecionadas para a análise multivariada e foram consideradas significativas nesta análise as variáveis com valor de $\mathrm{p}<0,05$. Os fatores associados à automedicação foram: utilizar medicamento influenciado por propaganda aumenta em 1,2 vezes a prevalência de automedicação em comparação a indivíduos que não se influenciam pela propaganda ( $\mathrm{RP}=1,2$; IC95\%=1,1-1,4); a prática de indicar algum medicamento para outras pessoas aumenta em 1,4 vezes a prevalência do indivíduo em se automedicar ( $\mathrm{RP}=1,4$; IC95\%=1,1-1,6); o estado de saúde autorreferido muito bom/bom diminui em 0,8 vezes a prevalência de automedicação comparado à saúde regular/ruim ( $\mathrm{RP}=0,8 ; \mathrm{IC} 95 \%=0,6-0,9)$ e ter realizado a última consulta médica há mais de um mês da data da entrevista aumenta em 1,5 vezes a prevalência de se automedicar $(\mathrm{RP}=1,5 ; \mathrm{IC} 95 \%=1,1-2,1)$ (Tabela 5$)$. 
Tabela 3. Perfil da automedicação na população avaliada, Ouro Preto, 2015/2016

$\begin{array}{lcc}\text { Classe medicamentosa utilizada } & \text { N } & \% \\ \text { Analgésicos/antitérmicos } & & \\ \text { Antigripais } & 123 & 65,8 \\ \text { anti-inflamatórios } & 40 & 21,4 \\ \text { Antialérgico } & 35 & 18,7 \\ \text { Chás medicinais } & 27 & 14,4 \\ \text { Vitaminas } & 21 & 11,2 \\ \text { Antiácidos e digestórios } & 19 & 10,2 \\ \text { Antibióticos } & 18 & 9,6 \\ \text { Anticoncepcionais } & 8 & 4,3 \\ \text { Laxantes } & 8 & 4,3 \\ \text { Outros } & 5 & 2,7 \\ \text { Vermífugos } & 3 & 1,6 \\ & 1 & 0,5\end{array}$

Sintoma (doença) que levou à utilização do medicamento não prescrito*

Dor de cabeça/febre $127 \quad 67,9$

$\begin{array}{lll}\text { Resfriado/gripe } & 61 & 32,6\end{array}$

$\begin{array}{lll}\text { Dores musculares } & 57 & 30,5\end{array}$

Cólica $\quad 41 \quad 21,9$

Dores de estômago/má digestão $\quad 28 \quad 15,0$

$\begin{array}{lll}\text { Alergia } & 25 & 13,4\end{array}$

$\begin{array}{lll}\text { Outros } & 17 & 9,1\end{array}$

$\begin{array}{lll}\text { Sinusite } & 13 & 6,9\end{array}$

Ansiedade/nervosismo/stress $\quad 10 \quad 5,3$

$\begin{array}{lll}\text { Vômito/diarria } & 7 & 3,7\end{array}$

Insônia $\quad 4 \quad 2,1$

Quem orientou o consumo?

$\begin{array}{lll}\text { Por conta própria } & 69 & 36,9\end{array}$

$\begin{array}{lll}\text { Mãe } & 60 & 32,1\end{array}$

Me baseei em prescrição médica anterior $\quad 21 \quad 11,2$

$\begin{array}{lll}\text { Balconista/farmacêutico } & 17 & 9,1\end{array}$

$\begin{array}{lll}\text { Vizinhos } & 14 & 7,4\end{array}$

Pai, irmãos e outros familiares $\quad 6 \quad 3,2$

Tempo após surgimento dos sintomas houve automedicação

$\begin{array}{lcr}\text { Entre } 1 \text { e } 6 \text { dias } & 159 & 85,0 \\ \text { Entre } 1 \text { semana e } 1 \text { mês } & 14 & 7,5 \\ \text { Mais de um mês } & 14 & 7,5\end{array}$

Reação adversa com a utilização desse(s) medicamento(s)

$\begin{array}{lll}\text { Não } & 178 & 95,2\end{array}$

Sim $\quad 9 \quad 4,8$

Satisfação com o uso desse(s) medicamento(s)

$$
\begin{array}{llll}
\text { Não } & 11 & 5,9
\end{array}
$$$$
\text { Sim } \quad 176 \quad 94,1
$$

Conhecimento se o uso desse(s) medicamento(s) pode causar algum problema

$\begin{array}{lll}\text { Não } & 97 & 51,9 \\ \text { Sim } & 90 & 48,1\end{array}$

Utilização de mais algum medicamento por ocasião da automedicação?

Não $152 \quad 81,3$

$\begin{array}{lrr}\text { Sim } & 35 & 18,7\end{array}$

*Dentre os 187 que utilizaram medicamentos não prescritos
Tabela 4. Motivos da automedicação entre a população avaliada, Ouro Preto, 2015/2016

\begin{tabular}{|c|c|c|}
\hline Motivo da automedicação & $\mathbf{N}$ & $\%$ \\
\hline Experiência anterior com esse medicamento & 102 & 54,5 \\
\hline $\begin{array}{l}\text { Sintomas muito simples, sendo desnecessário ir ao } \\
\text { médico }\end{array}$ & 91 & 48,7 \\
\hline Necessidade de uma melhora imediata & 69 & 36,9 \\
\hline Falta de tempo & 68 & 36,4 \\
\hline $\begin{array}{l}\text { É fácil e prático ir à farmácia e comprar o } \\
\text { medicamento }\end{array}$ & 44 & 23,5 \\
\hline $\begin{array}{l}\text { Utilização de sobras de medicamentos guardados } \\
\text { em casa }\end{array}$ & 33 & 17,6 \\
\hline Dificuldade para marcação de consulta médica & 29 & 15,5 \\
\hline $\begin{array}{l}\text { Compartilhamento do medicamento por alguém da } \\
\text { convivência }\end{array}$ & 21 & 11,2 \\
\hline $\begin{array}{l}\text { Falta de plano de saúde privado e/ou condição } \\
\text { financeira para pagar consulta }\end{array}$ & 14 & 7,5 \\
\hline Insatisfação em relação ao atendimento médico & 9 & 4,8 \\
\hline $\begin{array}{l}\text { Por influência da internet, TV ou outros meios de } \\
\text { comunicação }\end{array}$ & 8 & 4,3 \\
\hline Outro motivo & 3 & 1,6 \\
\hline
\end{tabular}

Tabela 5. Análise multivariada dos fatores associados ao uso de medicamentos não prescritos, Ouro Preto, 2015/2016

\begin{tabular}{lcc}
\multicolumn{1}{c}{ Variáveis } & $\begin{array}{c}\text { RP Ajustada } \\
\text { (IC 95\%) }\end{array}$ & p \\
$\begin{array}{l}\text { Uso de medicamento influenciado por } \\
\text { propaganda }\end{array}$ & & \\
$\quad$ Não versus Sim & $1,2(1,1-1,4)$ & 0,01 \\
Indicou ou indicaria algum medicamento & & \\
$\quad$ Não versus Sim & $1,4(1,1-1,6)$ & 0,00 \\
Percepção da própria saúde & & \\
$\quad \begin{array}{l}\text { Regular versus Muito boa/boa } \\
\text { Última consulta médica }\end{array}$ & $0,8(0,6-0,9)$ & 0,00 \\
$\quad$ Na última semana versus Entre 1 e 3 meses & $1,5(1,1-2,1)$ & 0,01 \\
$\quad$ Na última semana versus Há mais de 3 meses & $1,5(1,1-2,1)$ & 0,01 \\
\hline
\end{tabular}

\section{DISCUSSÃO}

No presente estudo foi observada uma elevada proporção de indivíduos que se automedicaram entre pessoas que tomaram medicamentos nos últimos 15 dias $(69,3 \%)$, sendo os medicamentos não prescritos mais utilizados os analgésicos/antitérmicos, seguidos pelos antigripais e os pelos anti-inflamatórios. Os fatores associados à automedicação foram o intervalo decorrido da última consulta médica, a utilização de medicamento influenciado por propaganda, a prática de indicar medicamento para outras pessoas e o estado de saúde autorreferido.

Em relação à alta prevalência de automedicação em adolescentes, outros estudos realizados no Brasil ${ }^{14,18,20,23}$ também observaram resultados elevados. Nestes estudos, os períodos recordatórios variaram de 15 dias até períodos não delimitados. Os trabalhos utilizando períodos recordatórios mais longos ${ }^{14,20,23}$ apresentaram prevalências mais elevadas, variando de $78,6 \%$ a 
96,2\%; entretanto, pode ter ocorrido uma superestimação do consumo devido ao uso acumulado de medicamentos.

No presente estudo, a experiência prévia foi o principal motivo para o uso de medicamentos sem prescrição. Os analgésicos/antitérmicos foram a classe farmacológica mais utilizada entre os medicamentos não prescritos, seguidos pelos antigripais e os anti-inflamatórios. É importante ressaltar que o consumo de analgésicos/antigripais/ anti-inflamatórios é favorecido pela facilidade de sua aquisição, uma vez que são medicamentos de venda livre e disponíveis em drogarias, farmácias domésticas ou, até mesmo, em supermercados ${ }^{24}$. Os analgésicos são medicamentos indicados para alívio da dor, o que também corrobora a sua grande utilização de forma não prescrita. Em outros trabalhos, os analgésicos também foram a classe farmacológica mais consumida por automedicaçãa ${ }^{14,18,20,25-28}$. De maneira geral, resfriado/gripe são processos autolimitados e não justificariam o uso de medicamentos antigripais. Entretanto, $85 \%$ dos indivíduos fizeram uso do medicamento não prescrito entre um e seis dias do início dos sintomas e $36,9 \%$ relataram utilizá-lo no intuito de uma melhora imediata, confirmando o objetivo do uso para alívio da sintomatologia. Também deve ser ressaltado um considerável consumo de antialérgico entre os indivíduos que se automedicaram, o que pode ser justificado pelas características climáticas da cidade de Ouro Preto, com elevada umidade do ar, propiciando o crescimento fúngico e levando, consequentemente, a uma maior frequência de doenças respiratórias alérgicas. Chama a atenção a ocorrência de oito casos de automedicação pelo uso de antibiótico. Atualmente segundo a RDC no 20, 2011 da Agência Nacional de Vigilância Sanitária (ANVISA) ${ }^{29}$, os antibióticos só podem ser adquiridos com receita médica. Então, possivelmente estas pessoas utilizaram sobras de antibióticos prescritos anteriormente ou a drogaria/farmácia dispensou o medicamento sem a apresentação da receita médica.

A cefaleia foi responsável pela maior parte dos casos de automedicação, semelhante ao encontrado em outros estudos ${ }^{14,25,30,31}$. A gripe e o resfriado foram a segunda causa mais relatada para a utilização de medicamento não prescrito, possivelmente, com o intuito de aliviar a sintomatologia com mais rapidez, como previamente discutido. Cefaleia, gripe e resfriado são doenças/sintomas nas/os quais são utilizados analgésicos e antitérmicos o que corrobora com a maior utilização observada desta classe medicamentosa na automedicação.

Quanto aos fatores que influenciaram na automedicação, observou-se que, apesar de a maioria dos participantes considerarem as propagandas de medicamentos pouco ou nada confiáveis do ponto de vista científico, $45,2 \%$ já utilizaram algum medicamento por influência da propaganda. Foi verificado que utilização de medicamento influenciado por propaganda aumenta em 1,2 vezes a prevalência de automedicação em comparação a indivíduos que não são influenciados. Isso confirma a influência da mídia em aspectos muito importantes do cotidiano dos indivíduos, como o uso de medicamentos. Apesar da regulamentação das propagandas de medicamentos pela ANVISA através da RDC 96/2008, é comum observar nos meios de comunicação propagandas de medicamentos que estão em desacordo com a referida legislação ${ }^{32}$. Almeida et al. ${ }^{7}$ também constataram que a maioria da população avaliada $(81,5 \%)$ confia pouco nas propagandas de medicamentos, mas, consistentemente, $68 \%$ nunca fizeram uso de um medicamento influenciados por estas.

Um fator associado negativamente com a prática da automedicação foi o estado de saúde autorreferido. O indivíduo que se autorrefere como tendo boa saúde certamente tenderá a não utilizar medicamentos, sejam estes prescritos ou não.

Um intervalo entre a última consulta e a data da entrevista superior a um mês aumenta em 1,5 vezes a prevalência de se automedicar, o que é um resultado esperado, uma vez que, se os indivíduos não procuram serviços de saúde há algum tempo, provavelmente utilizam medicamentos não prescritos para resolução de doenças/sintomas mais usuais. Também a prática de indicar o medicamento para outras pessoas foi um fator positivamente associado à automedicação. A prática de indicar medicamentos demonstra que o indivíduo considera desnecessária a consulta médica para determinados sintomas/doenças e sendo assim, maior será o seu risco de se automedicar.

Apesar de quase a totalidade dos entrevistados não apresentar reação adversa ao medicamento utilizado sem prescrição, mais da metade não sabia sobre os possíveis problemas que esse medicamento poderia lhes causar. Esses dados apontam para os riscos da automedicação em que o indivíduo consome fármacos sem adequado conhecimento sobre seus efeitos indesejados.

O presente estudo apresenta algumas limitações que devem ser pontuadas. Os estudos transversais não permitem inferir causalidade, uma vez que não consideram a variável tempo em sua análise, entretanto, fornecem informações relevantes que podem nortear estudos longitudinais. A utilização de uma amostra não probabilística também pode levar a viés, contudo, a proporção de indivíduos de cada categoria funcional foi mantida. Quanto à validade externa, provavelmente o estudo não representa a população de adolescentes como um todo, visto que no IFMG os alunos estão cursando um curso profissionalizante e, por passarem por um processo de seleção para ingressar na Instituição, podem não representar a população geral de adolescentes do município ou mesmo das escolas estaduais.

Sendo a população avaliada constituída em sua maioria por adolescentes, ações que visem à educação em saúde conscientizando sobre o uso racional de medicamentos podem minimizar as 
consequências relacionadas a este importante problema de saúde pública. Durante a realização desta pesquisa, e visando aumentar o conhecimento sobre medicamentos e sobre os riscos da sua utilização de forma incorreta, foi elaborado e distribuído à população um material com informações educativas sobre automedicação e uso racional dos medicamentos. A adolescência é uma fase caracterizada por transformações biopsicossociais na qual o indivíduo forma consciência crítica a respeito de vários assuntos e pode disseminar o conhecimento adquirido entre membros da família e outras pessoas de sua convivência.
A elevada proporção de indivíduos que se automedicaram entre pessoas que tomaram medicamentos nos últimos 15 dias nesta escola de ensino profissionalizante revela a importância da conscientização sobre os riscos da automedicação, especialmente dos adolescentes que se encontram formando opinião acerca de vários assuntos. Assim, trabalhos que promovam, durante o seu desenvolvimento, ações de conscientização desta população são de grande importância, na medida em que contribuem para a formação de cidadãos cientes da sua parcela de responsabilidade para o uso racional de medicamentos.

\section{REFERÊNCIAS}

1. Paulo LG, Zanini AC. Automedicação no Brasil. AMB Rev Assoc Med Bras. 1988;34(2):69-75. PMid:3244846.

2. Vilarino JF, Soares IC, Silveira CM, Rödel AP, Bortoli R, Lemos RR. Perfil da automedicação em município do sul do Brasil. Rev Saude Publica. 1998;32(1):43-9. http://dx.doi.org/10.1590/S0034-89101998000100006. PMid:9699344.

3. Sistema Nacional de Informações Tóxico-Farmacológicas. Evolução dos casos registrados de intoxicação humana por agente tóxico [Internet]. Rio de Janeiro: Fundação Oswaldo Cruz; 2009 [citado em 2017 set 20]. Disponível em http://sinitox.icict.fiocruz.br/dados-nacionais

4. Agência Nacional de Vigilância Sanitária. Parcerias para diminuir o mau uso de medicamentos. Rev Saude Publica. 2006;40(1):191-2. http://dx.doi. org/10.1590/S0034-89102006000100029. PMid:16411002.

5. World Health Organization. The benefits and risks of selfmedication. WHO Drug Inf. 2000;14:1-2.

6. Figueiredo MC, Kothe V, Vieira L, Emerim J, Silva KVCL. Armazenagem e descarte de medicamentos: uma questão de educação e saúde. In: Anais do $3^{\circ}$ Congresso Internacional de Tecnologias para o Meio Ambiente [Internet]; 2012 abril 25-27; Bento Gonçalves, BR. Bento Gonçalves: Proamb; 2012. [citado em 2017 set 18]. p. 1-8.Disponível em: http://www.proamb.com. br/downloads/befr4g.pdf

7. Almeida C, Souza DO, Ferreira MB, Wofchuk S. Levantamento do uso de medicamentos por estudantes do ensino médio em duas escolas de Porto Alegre, RS, Brasil. Ciênc Educ (Bauru). 2012;18(1):215-30. http://dx.doi. org/10.1590/S1516-73132012000100013.

8. Lunde PKM, Baksaas I. Epidemiology of drug utilization: basic concepts and methodology. Acta Med Scand Suppl. 1988;721(Suppl):7-11. PMid:3281414.

9. Loyola AIF, Uchoa E. Automedicação, motivações e características de sua prática. Rev Med Minas Gerais. 2002;12(4):219-27.

10. Musial DC, Dutra JS, Becker TCA. A automedicação entre os brasileiros. Rev Saude e Biol. 2007;2(2):5-8.

11. Aquino DS. Por que o uso racional de medicamentos deve ser uma prioridade? Cien Saude Colet.2008;13(Supl):733-6.http://dx.doi.org/10.1590/S141381232008000700023

12. Barros ARR, Griep RH, Rotenberg L. Self medication among nursing workers from public hospitals. Rev Lat Am Enfermagem. 2009;17(6):101522.http://dx.doi.org/10.1590/S0104-11692009000600014. PMid:20126945.
13. Silva RCG, Oliveira TM, Casimiro TS, Vieira KAM, Tardivo MT, Faria M Jr, Restini CBA. Automedicação em acadêmicos do curso de medicina. Medicina (Ribeirão Preto). 2012;45(1):5-11

14. Rios MF, Souza WA, Siqueira VMS, Podesta MHMC, Melo GGP, Zuba AG, et al. Perfil da automedicação dos alunos de uma escola técnica do sul de Minas Gerais. Rev Univ Vale Rio Verde. 2013;11(2):420-31.http:// dx.doi.org/10.5892/ruvrd.v11i2.420431

15. Pereira AC Jr, Telles PCP Fo, Azevedo DSS. Automedicação: consumo, orientação e conhecimento entre acadêmicos de enfermagem. Rev enferm UFPE on line. 2013;7(6):4472-8. http://dx.doi.org/10.5205/reuol.416433013-1-SM.0706201321

16. Martins LB, Costa-Paiva L, Osis MJ, Sousa MH, Pinto AM No, Tadini V. Conhecimento sobre métodos anticoncepcionais por estudantes adolescentes. Rev Saude Publica. 2006;40(1):57-64. http://dx.doi.org/10.1590/S003489102006000100010. PMid:16410983.

17. Santos AF, Mendonça PMH, Santos LA, Silva NF, Tavares JKL. Anabolizantes: conceitos segundo praticantes de musculação em Aracajú (SE). Psicol Estud. 2006;11(2):371-80. http://dx.doi.org/10.1590/S1413-73722006000200016.

18. Pereira FSVT, Bucaretchi F, Stephan C, Cordeiro R. Automedicação em crianças e adolescentes. J Pediatr. 2007;83(5):453-8. http://dx.doi.org/10.2223/ JPED.1703.

19. Silva IM, Catrib AM, de Matos VC, Gondim AP. Automedicação na adolescência: um desafio para a educação em saúde. Cien Saude Colet. 2011;16(1Supl):1651-60. http://dx.doi.org/10.1590/S1413-81232011000700101. PMid:21503517.

20. Pardo IMCG, Jozala DR, Carioca AL, Nascimento SRD, Santucci VCR. Automedicação: prática frequente na adolescência? Estudo em uma amostra de estudantes do ensino médio de Sorocaba. Rev Fac Cienc Med Sorocaba. 2013;15(2):11-5

21. Bertoldi AD, Barros AJD, Hallal PC, Lima RC. Utilização de medicamentos em adultos: prevalência e determinantes individuais. Rev Saude Publica. 2004;38(2):228-38. http://dx.doi.org/10.1590/S0034-89102004000200012. PMid:15122379.

22. Associação Brasileira de Empresas de Pesquisa [Internet].Critério Brasil 2018.São Paulo: ABEP; 2010 [citado em 2017 set 18]. (Vol. 2010). Disponível em: http://www.abep.org/criterio-brasil

23. Mayolo T, Fernandes LC. Análise da prática de automedicação em uma drogaria de Arroio do Meio-RS. Revista Destaques Acadêmicos.2012;4(3):7-18. 
24. Silva CH, Giugliani ERJ. Consumo de medicamentos em adolescentes escolares: uma preocupação. J Pediatr. 2004;80(4):326-32. http://dx.doi. org/10.2223/1208.

25. Arrais PSD, Coelho HLL, Batista MCDS, Carvalho ML, Righi RE, Arnau JM. Perfil da automedicação no Brasil. Rev Saude Publica. 1997;31(1):71-7. http://dx.doi.org/10.1590/S0034-89101997000100010. PMid:9430928.

26. Loyola AI Fo, Uchoa E, Guerra HL, Firmo JO, Lima-Costa MF. Prevalência e fatores associados à automedicação: resultados do projeto Bambuí. Rev Saude Publica. 2002;36(1):55-62. http://dx.doi.org/10.1590/S003489102002000100009. PMid:11887230.

27. Arrais PS, Fernandes ME, Pizzol TD, Ramos LR, Mengue SS, Luiza VL, et al. Prevalence of self-medication in Brazil and associated factors. Rev Saude Publica. 2016;50(2Suppl):13s. http://dx.doi.org/10.1590/s15188787.2016050006117. PMid:27982373.

28. Domingues PHF, Galvão TF, Andrade KRC, Araújo PC, Silva MT, Pereira MG, et al. Prevalência e fatores associados à automedicação em adultos no Distrito Federal: um estudo transversal de base populacional. Epidemiol Serv Saude. 2017;26(2):319-30. http://dx.doi.org/10.5123/ S1679-49742017000200009. PMid:28492773.
29. Brasil. Ministério da Saúde. Agência Nacional de Vigilância Sanitária. Resolução - RDC n²0, de 05 de maio de 2011. Dispõe sobre o controle de medicamentos à base de substâncias classificadas como antimicrobianos, de uso sob prescrição, isoladas ou em associação. Diário Oficial da União [Internet], 09 de maio de 2011 [citado em 2017 set 18]. Disponível em: http://www.anvisa.gov.br/sngpc/Documentos2012/RDC\%2020\%202011. pdf

30. Vitor RS, Lopes CP, Menezes HS, Kerkhoff CE. Padrão de consumo de medicamentos sem prescrição médica na cidade de Porto Alegre, RS. Cien Saude Colet.2008;13(Supl):737-43. PMid:21936179.http://dx.doi. org/10.1590/S1413-81232008000700024.

31. Gonçalves D, Santos BRM, Gonçalves ML, Aragão CCV, Gatti RM, Yavo B. Prática de automedicação entre usuários de uma farmácia-escola. Rev Bras Cien Saude. 2009;7(22):23-32.

32. Silva RB, Corte TWF. A propaganda de medicamentos e sua adequação conforme a RDC 96/2008. Revista da Graduação. 2009;2(2):1-11.

Recebido em: Set. 27, 2017

Aceito em: Mar. 31, 2018 